ORIGINAL RESEARCH

\title{
Interfang Distances of Rattlesnakes: Sexual, Interspecific, and Body Size-related Variation, and Implications for Snakebite Research and Management
}

\author{
William K. Hayes, PhD; Aaron G. Corbit, PhD; Michael D. Cardwell, MS; Shelton S. Herbert, PhD \\ From the Department of Earth and Biological Sciences, Loma Linda University, Loma Linda, CA (Drs Hayes, Corbit, and Herbert, and Mr \\ Cardwell); the Department of Biology, Southern Adventist University, Collegedale, TN (Dr Corbit); the Department of Biology, San Diego State \\ University, San Diego, CA (Mr Cardwell); and the Department of Biology, Pacific Union College, Angwin, CA (Dr Herbert).
}

\begin{abstract}
Objectives.-Snakebite severity corresponds to size of snake because the amount of venom a snake injects is positively associated with snake size. Because fang marks are often present on snakebite patients, we tested whether the relationship between snake length and distance between fang puncture wounds can be generalized for rattlesnakes of genus Crotalus.

Methods.-We measured 2 interfang distances from 79 rattlesnakes of both sexes, 5 species, and varying body length: 1) distance between fang bases in anesthetized snakes, and 2) distance between fang punctures in a membrane-covered beaker bitten defensively.

Results. - Statistical analyses supported our 2 hypotheses, that 1) body size-related fang divergence during fang protraction (ie, anterolateral movement during fang erection), and 2) the relationship between snake length and interfang distance are similar between the sexes and among different rattlesnake species. We therefore derived a general equation to estimate snake length based on distance between fang marks, and recommended 5 snake size categories: very small $(<10 \mathrm{~mm})$, small $(10-15$ $\mathrm{mm})$, medium $(15-20 \mathrm{~mm})$, large $(20-25 \mathrm{~mm})$, and very large $(>25 \mathrm{~mm})$.

Conclusions. - The distance between fang marks on a snakebite patient may be used to estimate the size or size category of the offending snake, which in some cases may have predictive value for overall clinical severity of a given envenomation. Assessing interfang distance from puncture wounds can improve snakebite research and anticipation of snakebite severity.
\end{abstract}

Keywords: animals, body size, Crotalus, envenomation, snakebite treatment, venom

\section{Introduction}

Several factors potentially contribute to the clinical severity of a venomous snake bite. These have been categorized as factors related to the human victim and those related to the snake. ${ }^{1}$ Factors related to the patient include body mass, anatomical location of the bite, presence of clothing, delay to treatment, and the treatment itself, whereas those related to the snake include species (eg, venom composition and toxicity) and body size (eg, fang length and amount of

Corresponding author: William K. Hayes, PhD, Department of Earth and Biological Sciences, Loma Linda University, Loma Linda, CA 92350; e-mail: whayes@1lu.edu.

Submitted for publication July 2016.

Accepted for publication March 2017. venom injected). Of these factors, size of the snake (along with size of the patient) may have the greatest effect on envenomation severity. Hospital-based studies have consistently shown that larger snakes cause more severe bites ${ }^{2-6}$ because they inject larger quantities of venom. ${ }^{7-10}$ Knowing the size of the envenoming snake has significant predictive value for overall clinical severity, symptom progression, and the amount of antivenom needed to treat a bite. ${ }^{4-6}$

Since knowledge of the size of the envenoming snake may be of use to clinicians, and because the perceived size of the snake reported by patients or other witnesses may be unreliable (or unavailable if the patient is unconscious), a more objective means of determining snake size in a clinical setting would be desirable. One potential method is to use the distance between the 
wounds caused by the fangs (ie, fang spread or interfang distance) to estimate snake size. ${ }^{11}$ Zamudio et $\mathrm{al}^{12}$ demonstrated experimentally that the interfang distance, measured by strikes to a flat foam surface, predicted total body length in the Western Diamondback Rattlesnake (Crotalus atrox). Janes et $\mathrm{al}^{4}$ found that in patients bitten by southern California rattlesnakes (genus Crotalus), snakes in the larger size classes had larger measurable interfang distances. Thus, in cases wherein 2 fang marks are present, the treating physician should be able to infer the relative size and danger of the offending snake.

The main purpose of this study was to evaluate whether the relationship between snake length and distance between fang puncture wounds is similar between the sexes and among different species of medically significant venomous snakes in southern California. Because head size relative to body size varies somewhat among different species of rattlesnakes, and even to some extent between the sexes, ${ }^{13,14}$ differences in the relationship might exist. When rattlesnakes protract their fangs to deliver a bite (ie, extend them from a resting, or retracted, position against the roof of the mouth), the fang tips diverge, which increases the distance between them. ${ }^{12,15}$ This kinematic feature of anterolateral fang movement may also differ between the sexes and among species. Collectively, these considerations prompted us to experimentally test 2 hypotheses using 5 species of southern California rattlesnakes: 1) that body size-related fang divergence during fang protraction associated with a defensive bite is similar between the sexes and among different rattlesnake species; and 2) that the relationship between snake length and distance between fang punctures during a defensive bite is similar between the sexes and among different rattlesnake species. Support for the latter hypothesis in particular would suggest that a single equation or set of measurements could be used by researchers and treating physicians to estimate or categorize a rattlesnake's body length - and relative danger from a bite-by measuring the distance between 2 fang punctures when visible on a snake-bitten patient.

\section{Methods}

\section{SUBJECTS}

We obtained measurements from 5 southern California rattlesnake species of varying snout-vent length (SVL). The 79 specimens included 16 Crotalus cerastes (8 male, 4 female, 4 undetermined sex; $31-52 \mathrm{~cm}$ SVL), 20 C (oreganus) helleri (14 male, 6 female; 41$105 \mathrm{~cm}$ ), $12 C$ (mitchellii) pyrrhus (5 male, 7 female; $32-91 \mathrm{~cm}), 10$ C ruber (8 male, 2 female; $53-123 \mathrm{~cm}$ ), and $21 C$ scutulatus ( 8 male, 13 female; $30-83 \mathrm{~cm}$ ). We chose these species because they represent diverse clades within the genus ${ }^{16}$ and vary substantially in adult size, ${ }^{13}$ both of which might affect body proportions. These species also inflict a substantial number of bites in southern California, where clinicians often treat bites from multiple species. ${ }^{2-6}$ Our sample included both long-term captives and recently captured specimens, all from southern California locations with the exception of $2 \mathrm{C}$ cerastes from Arizona. We assumed that husbandry exerted a negligible effect on the kinematics of fang use, and therefore included all snakes in our analyses. All subjects appeared to be in excellent health.

\section{MEASUREMENTS}

To minimize handling and disturbance of snakes, we obtained data from each specimen in a single session. First, we determined interfang distance during a defensive bite (ie, fangs protracted) by subjecting each snake to a routine venom extraction, which involved grasping the snake by the head and inducing it to voluntarily bite a Ziploc plastic sandwich bag secured by a rubber band over the top of a glass beaker (50 or $150 \mathrm{~mL}$, depending on size of snake). We then used a caliper to measure the distance (nearest $0.5 \mathrm{~mm}$ ) between the pair of fang punctures in the membrane. Next, we anesthetized the snake in a transparent plastic tube using sevoflurane ${ }^{17}$ and used a caliper to measure interfang distance (nearest $0.5 \mathrm{~mm}$ ) between the midlines of the bases of the fang sheaths. ${ }^{12}$ Finally, we measured the snout-vent length (nearest $1 \mathrm{~cm}$ ) of the anesthetized snake with a metal ruler and determined its sex via sexing probes. Research was conducted with permits issued by the California Department of Fish and Wildlife and approval by the Institutional Animal Care and Use Committee of Loma Linda University.

\section{ANALYSES}

We conducted 3 sets of analyses using SPSS 13.0 (SPSS Inc, Chicago, IL), with standard defaults and $\alpha$ set at 0.05 . Prior to analyses, we $\log _{10}$-transformed the 2 measures of interfang distance (retracted fang bases and protracted fang punctures) to meet multivariate assumptions of normality, homoscedasticity, and linearity. Because of missing data for sex $(\mathrm{n}=4 C$ cerastes $)$ and distance between fang bases ( $\mathrm{n}=1 C$ scutulatus), our sample size varied from $n=74-79$, depending on which variables were included in a given model.

First, we compared the sexes and 5 species using a multivariate analysis of covariance (MANCOVA ${ }^{18}$ ) model that included both measures of interfang distance (retracted fang bases and protracted fang punctures) as dependent 
variables and SVL as a covariate. We tested the assumption of homogeneity of regression slopes by including interaction terms between the covariate and the 2 independent variables (sex, species), and then removed those interactions from the final model because they were nonsignificant. We followed up the MANCOVA model with univariate analyses of covariance $\left(\mathrm{ANCOVAs}{ }^{18}\right.$ ) that tested each dependent variable separately, likewise testing for the homogeneity of regression slopes, and used Bonferroni multiple comparisons to test for group (species) differences. We computed effect sizes for the MANCOVA and ANCOVAs as multivariate partial $\eta^{2}$ and partial $\eta^{2}$, respectively, which roughly correspond to percent variance explained, with values of $\sim 0.01$, $\sim 0.06$, and $\geq 0.14$ loosely regarded as small, moderate, and large effects, respectively. ${ }^{19}$ Because $\eta^{2}$ is upwardbiased when multiple predictors are included in a model, ${ }^{20}$ the values for main effects and interactions in our models always summed to $>1.0$; thus, we adjusted these values by dividing each by the sum of all multivariate partial $\eta^{2}$ values. ${ }^{21}$

Second, we conducted a mixed ANCOVA ${ }^{18}$ to better characterize the relationship between the 2 measures of interfang distance (distances between fang bases and fang punctures). This model included measurement method as a within-subjects factor, species as a between-subjects factor, and snake length as a cofactor for explaining interfang distance. Again, we initially included the interaction terms between the covariate and the 2 independent variables (measurement method, species), and then removed those interactions from the final model because they were nonsignificant. We excluded sex from the model to avoid the noise of a trivial factor. We again computed adjusted partial $\eta^{2}$ as an effect size.

Third, we used a simple regression $\operatorname{model}^{18}$ to describe the relationship between distance between fang punctures and SVL for both sexes and all snake species combined. This analysis provided an equation with coefficients that could be used to estimate, in reverse, the size of a snake based on the distance between the fang punctures of a snakebite victim. We used $r^{2}$ as an effect size, interpreted as percent variance explained, with values of $\sim 0.01, \sim 0.09$, and $\geq 0.25$ loosely deemed as small, moderate, and large effects, respectively. ${ }^{19}$ To compare effect sizes for the 2 measures of interfang distance, we ran a similar regression for distance between fang bases.

\section{Results}

The MANCOVA model revealed a highly significant effect of snake length on the combined dependent variables of distance between fang bases and distance between fang punctures (Wilks' $\Lambda=0.159, F_{2,62}=$ 163.76, $P<.001$, adjusted multivariate partial $\eta^{2}=$ 0.77). As expected, interfang distance increased substantially with snake length, and with an exceptionally large effect size. Although interfang distances were similar for the 2 sexes (Wilks' $\Lambda=0.967, F_{2,62}=1.056, P=.35$, adjusted multivariate partial $\left.\eta^{2}=0.03\right)$, they differed significantly among the 5 species (Wilks' $\Lambda=0.711$, $F_{8,124}=2.88, P=.008$, adjusted multivariate partial $\eta^{2}$ $=0.14)$. No interactions among sex, species, and snake length were significant.

The 2 univariate ANOVAs conducted as a follow-up to the MANCOVA indicated that species differences were significant for distance between fang bases $\left(F_{4,63}=\right.$ 5.48, $P=.001$, adjusted partial $\left.\eta^{2}=0.21\right)$, but not for distance between fang punctures $\left(F_{4,63}=0.70, P=.60\right.$, adjusted partial $\eta^{2}=0.03$; note the substantially smaller effect size). Otherwise, results for both models were similar to the MANCOVA in that the effect of sex was negligible (adjusted partial $\eta^{2} \leq 0.01$ ) and snake length remained highly significant (adjusted partial $\eta^{2}=0.27-$ 0.28). Again, no interactions were significant. Species rankings of relative distance between the fang bases (from greatest to smallest value), and estimated marginal means (inverse-transformed from $\log _{10}$ values) at 65.9 $\mathrm{cm}$ SVL for all taxa, were $C$ cerastes $(8.0 \mathrm{~mm})$, C scutulatus $(7.9 \mathrm{~mm}), C$ helleri $(7.8 \mathrm{~mm}), C$ pyrrhus $(7.3 \mathrm{~cm})$, and $C$ ruber $(6.3 \mathrm{~cm})$. Bonferroni multiple comparisons indicated that the distance between fang bases was significantly less for $C$ ruber than those of $C$ cerastes, $C$ scutulatus, and $C$ helleri, and that all other species comparisons were similar.

The mixed ANCOVA model confirmed a highly significant difference between the 2 measures of interfang distance $\left(F_{1,72}=83.68, P<.001\right.$, adjusted partial $\eta^{2}=0.34$; Figure). Controlling for snake length and species (and disregarding sex) in the model, the estimated marginal mean (for all snakes) at $64.3 \mathrm{~cm}$ SVL was $7.4 \mathrm{~cm}$ for distance between fang bases and $14.3 \mathrm{~cm}$ for distance between fang punctures. Thus, during fang protraction, the fang spread increased by a mean of $93.2 \%$ compared with the retracted state. Species rankings of percentage increase in fang spread (from highest to lowest, with mean $\pm 1 \mathrm{SE}$ ) was $C$ ruber (118.7 $\pm 20.1 \%)$, C pyrrhus $(102.0 \pm 10.8 \%), C$ helleri $(100.3 \pm 8.8 \%), C$ cerastes $(87.2 \pm 7.3 \%)$, and $C$ scutulatus $(83.6 \pm 5.9 \%)$.

The regression model that described distance between fang punctures in relation to snake length for all snakes (both sexes and all species) provided a highly significant fit $\left(F_{1,77}=200.54, P<.001\right)$ with an exceptional effect size $\left(r^{2}=0.72\right)$. The model, with 


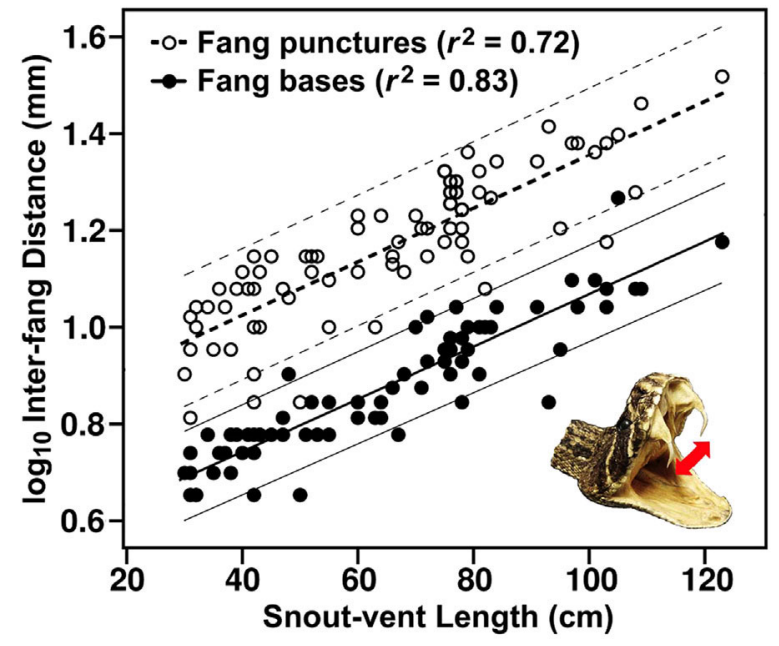

Figure. Relationships between snout-vent length and 2 measures of interfang distance for southern California rattlesnakes (genus Crotalus): distance between the midline of the fang sheaths (fang bases) measured in anesthetized snakes, and distance between fang marks in a plastic membrane from a defensive bite obtained during a venom extraction (fang punctures). Data are pooled for the 2 sexes and 5 species tested due to statistical similarity. 95\% CI are illustrated. See text for regression equations (Equations 1 and 2). $\mathrm{n}=79$ snakes.

standard error (1 SE) for coefficients shown parenthetically, was in the form:

$\log _{10}$ Fang puncture distance $=0.006( \pm 0.000) \mathrm{SVL}+$

$$
\mathbf{0 . 8 0 3}( \pm 0.027)
$$

The similar regression for distance between fang bases revealed a stronger relationship $\left(F_{1,76}=375.10, P<\right.$ $\left..001, r^{2}=0.83\right)$ in the form:

$$
\begin{aligned}
& \log _{10} \text { Fang base distance }=0.005( \pm 0.000) \mathrm{SVL}+ \\
& \mathbf{0 . 5 2 6}( \pm 0.019)
\end{aligned}
$$

\section{Discussion}

In this study, we experimentally tested 2 hypotheses regarding the relationship between rattlesnake body size and interfang distance to provide useful information to researchers and treating physicians regarding the relative size of a snake that has bitten a patient. We measured distances between the 2 fangs at the retracted (resting) and protracted (extended) states, and compared these measurements between the sexes, among 5 species, and among different sizes of rattlesnakes.

\section{GENERALITY OF THE SNAKE SIZE-INTERFANG DISTANCE RELATIONSHIP}

The results supported our first hypothesis, that size-related fang divergence during fang protraction associated with a defensive bite is similar between the sexes and among different rattlesnake species. We conclude this based on the lack of interactions between snake length and sex and between snake length and species in our MANCOVA and ANCOVA models. (Supplemental ANCOVAs, not shown, confirmed the absence of sex, interspecific, and ontogenetic differences in actual measurements of percentage of fang divergence.) During protraction (elevation) of the fangs from their retracted (resting) position, the distance between the fangs (and presumably the fang tips within the fang sheaths, although we did not know their exact position relative to the fang bases at resting position) increased nearly 2 -fold, averaging $93.2 \%$. This kinematic feature of the fangs, the anterolateral rotation of the fangs first described by Mitchell, ${ }^{15}$ may serve several functions: it should protect the snake from fang penetration of its lower jaw during a bite ${ }^{15}$; it should increase the likelihood of successful prey capture and envenomation (making contact and penetrating soft tissue rather than hitting bone) when biting a fast-moving prey item $^{22}$ (the strike is generally aimed at the prey's head ${ }^{23}$ ); and it might facilitate intraoral transport during prey consumption. ${ }^{24,25}$

The results also supported our second hypothesis, that the highly significant relationship between snake length and distance between fang punctures during a defensive bite is similar between the sexes and among different rattlesnake species. However, the 2 measures of interfang distance contrasted with regard to interspecific variation, which existed for the fang bases but was absent for the fang punctures. Interspecific differences in the relationship between snake length and distance between fang bases likely result from interspecific variation in head shape. ${ }^{13}$ The fact that interspecific differences disappear once the fangs become protracted probably results from the flexible nature of the skull (ie, cranial kinesis ${ }^{22,26}$ ) that increases variability in the distance between the protracted fang tips. Variation in the relationship was clearly greater for fang punctures $\left(r^{2}=0.72\right)$ than for fang bases $\left(r^{2}=0.83\right)$, and this despite the pliable heads of anesthetized snakes that, in our opinion, caused greater measurement error for distance between fang bases than for distance between fang punctures. Zamudio et $\mathrm{al}^{12}$ obtained a higher coefficient of determination for fang bases in rigid, preserved specimens of $C$ atrox $\left(r^{2}=0.90\right)$, but did not report a value for fang punctures associated with defensive bites. de Roodt et $\mathrm{al}^{10}$ reported an $r^{2}$ of 0.83 for the distance between the midpoint of erected fangs and body length in $C$ durissus terrificus.

Our findings suggest that a single equation or set of measurements could be used by researchers and clinicians to estimate or categorize snake length by 
measuring the distance between fang puncture wounds on a snake-bitten patient. This information is particularly valuable because knowledge of the snake's size can benefit researchers who study snakebite management, and can help the treating physician anticipate overall clinical severity, symptom progression, and the amount of antivenom needed to treat a bite. ${ }^{6}$ Our basic equation that derived fang puncture distance from snake length (Equation 1) can be algebraically rearranged to predict snake length from fang puncture distance:

Snake length $(\mathrm{cm})=\frac{\log _{\mathbf{1 0}} \text { Fang spread }(\mathrm{mm})-0.803}{0.006}$

$$
\begin{aligned}
& \text { Snake length }(\mathrm{cm})=\left(( 1 6 6 . 6 7 ) \left(\log _{10}\right.\right. \text { Fang spread } \\
& (\mathrm{mm})))-133.83
\end{aligned}
$$

Since treating physicians rarely see the offending snake, and because eyewitness descriptions of the snake have limited reliability, snake size can still be estimated or categorized objectively when 2 fang marks are present. To facilitate use in a clinical setting, we offer simple guidelines in the Table for using interfang puncture distances to categorize snake size. The snake size classes begin with an easily remembered $<10 \mathrm{~mm}$ interfang distance (very small snake), and continue through 5-mm increments (small, medium, and large snakes) to $>25 \mathrm{~mm}$ (very large snake).

\section{LIMITATIONS}

Our conclusions, general equation, and guidelines in the Table should be applied only to rattlesnakes of the genus Crotalus. Three additional genera of dangerous and highly venomous snakes occur in the United States: rattlesnakes of the genus Sistrurus, copperheads and cottonmouths of the genus Agkistrodon, and coral snakes of the genus Micrurus. Zamudio et $\mathrm{al}^{12}$ found that the cottonmouth (Agkistrodon piscivorus) has a significantly greater interfang distance at equivalent body length than
$C$ atrox. Studies similar to ours are needed to ascertain the relationships between snake size and interfang distance for snakes of these other genera.

The relationship between snake length and interfang distance has been explored in other species of the genus Crotalus. Zamudio et $\mathrm{al}^{12}$ reported a $112 \%$ increase in distance between the fangs during fang protraction in defensive strikes by $C$ atrox, and Cundall $^{22}$ reported a $120 \%$ (neonates) and 130\% (adults) increase (apparently based on videotape analysis rather than actual fang punctures) in predatory strikes by $C$ horridus. These values are similar to or greater than those we measured in individual species (83.6\% to $118.7 \%$ ) and in all species pooled (93.2\%). Our results suggest that species differences within Crotalus are minimal given the small effect sizes, but differences among studies could result from the greater fang protraction that might occur during a full strike observed in other studies compared with the bites that we induced, which were devoid of a strike. Many snakebites undoubtedly result from an envenoming strike, but because viperid snakes in general are reluctant to bite defensively, and often do so only after the human contacts or grabs the snake, ${ }^{27,28}$ many bites also result absent a full strike.

For rattlesnakes of genus Crotalus, we are confident that our equation and the guidelines of the Table can predict snake length for most of the 21 recognized species that occur in North America north of Mexico, and probably most of the 45 recognized species in the New World. ${ }^{29}$ The 5 species we examined represented 3 of the $5^{30}$ or $7^{31}$ mitochondrial DNA clades (species groups) within the genus. We did not assess the small, montane, Mexican species of the $C$ intermedius, $C$ triseriatus, and $C$ stejnegeri clades, which generally have relatively small heads and possibly different body length-interfang distance relationships, but rarely inflict bites. Nor did we study the mostly large species that comprise the $C$ durissus clade, including $C$ adamanteus and $C$ horridus in the eastern United States, which have relatively longer but narrower heads, ${ }^{13}$ and might also have different body

Table. Recommended benchmarks for interpreting relative snake size (corresponding to anticipated envenomation severity) from the distance measured between 2 fang punctures in a patient bitten by a rattlesnake of the genus Crotalus

\begin{tabular}{lcc}
\hline Distance between fang punctures $(\mathrm{mm})$ & Approximate snout-vent length of rattlesnake $(\mathrm{cm})$ & Relative size of rattlesnake \\
\hline & $<33$ & Very small \\
$10-15$ & $33-62$ & Small \\
$15-20$ & $62-83$ & Medium \\
$20-25$ & $83-99$ & Large \\
$>25$ & $>99$ & Very large
\end{tabular}

Because of variation in interfang distance resulting from head size variation, cranial kinesis, and length of trunk extension associated with biting, actual snake lengths are imprecisely inferred from Equation 3 or 4, and therefore general size categories should be used by researchers. 
length-interfang distance relationships. The importance of phylogenetic variation in relative head size and its effect on interfang distance will depend on whether the goal is to estimate snake length or the capacity to deliver a large dose of venom. For estimating snake length, we strongly recommend the use of general body size categories (eg, small, medium, large $)^{4,6}$ because of inherent variation in the distance between puncture wounds resulting from head shape variation, cranial kinesis, and distance of trunk extension associated with biting. For estimating the capacity to deliver a large dose of venom, the interfang distance can be used directly without conversion to snake length. Compared with snake length, the interfang distance likely corresponds more closely to head size, quantity of venom available in the glands, and the amount of venom potentially injected during a bite, and therefore the interfang distance may be a more relevant measure.

Not all snakebites result in 2 discernable fang punctures. Fang marks are usually present in viperid bites, with patients exhibiting them in $100 \%$ of 100 cases $^{32}$ and $100 \%$ of 227 cases $^{33}$ in southern California; $100 \%$ of 24 cases in the western United States ${ }^{34} ; 100 \%$ of 28 cases in West Virginia (mostly involving Agkistrodon), ${ }^{35}$ and 68\% of 107 cases in North Carolina (mostly involving Agkistrodon). ${ }^{36}$ Fangs can even penetrate clothing, although reduced venom delivery may occur. ${ }^{37}$ In some cases, only a single puncture may be found from a unilateral (single-fang) bite, or 3 or more punctures may result from multiple bites. Unfortunately, the exact number of fang marks has seldom been quantified, although 2 or more fang punctures were present in $87.5 \%$ of 24 rattlesnake bites in the western United States, ${ }^{34}$ and in $57.6 \%$ of 33 Brazilian viper bites, primarily by the genus Bothrops. ${ }^{38}$ For 3 or more fang marks, discerning which punctures result from paired snake fangs in a double-bite scenario could pose a challenge.

Finally, the distance between fang punctures in a snakebite patient may increase as a result of swelling. The extent to which this might affect the distance between fang marks has not been quantified, and may be subject to interpretation by the physician. However, paired measurements of distances between fang punctures and length of the offending snakes $(n=39)$ in actual snakebites ${ }^{4}$ yielded values similar to those we provide in the Table.

\section{Conclusions}

Results from our experimental study supported our main hypothesis regarding the lack of sex and interspecific differences in the relationship between snake length and distance between the fang punctures resulting from defensive bites by rattlesnakes of the genus Crotalus. Because of the robustness and generality of this relationship, as inferred from our analyses, we have produced a general equation (in 2 algebraic forms, Equations 3 and 4) and simple guidelines in the Table for how researchers and physicians can estimate snake size from the distance between fang punctures on a snakebite victim.

Researchers should measure the distance between fang marks on snakebitten patients to estimate snake size and control for its effect when studying envenomation management. And given the importance of snake size in predicting overall clinical severity, symptom progression, and the amount of antivenom needed to treat a bite, ${ }^{4,6}$ treating physicians should measure the interfang distance to better anticipate symptom progression and antivenom needs. We emphasize the value of interfang distances only as a research tool and for anticipating the severity of a bite, because decisions on antivenom administration must be based entirely on the clinical effects (symptoms, signs, and laboratory abnormalities) observed. When ambiguity exists regarding physical evidence of snake size (ie, interfang distance), we urge the clinician to carefully query the snakebite victim or other eyewitnesses to gain a better understanding of the size of the snake and potential for severe envenomation.

Acknowledgments: We thank the following individuals for supplying or giving us access to snakes: Joe Banashek, Ronald Carter, Eric Dugan, Matt Goode, Bob Montoya, Curtis Rehling, Chris Rodriguez, Doug Stewart, Mike Wilcox, and Fritz Wuttke. The following individuals graciously assisted with data collection: Aliex Ferrer, Jessica Hayes Kuzan, and Neville Trimm. The reviewers, section editor, and interim editor-in-chief provided excellent suggestions that improved the manuscript.

Author Contributions: Study concept and design (WKH, MDC); obtaining funding (WKH, MDC); acquisition of the data (WKH, MDC, $\mathrm{SSH}$ ); analysis of the data (WKH, AGC); drafting of the manuscript (WKH, AGC); critical revision of the manuscript (WKH, AGC, MDC, $\mathrm{SSH}$ ); approval of final manuscript (WKH, AGC, MDC, SSH).

Financial/Material Support: Research was supported by the Loma Linda University Department of Earth and Biological Sciences and by out-of-pocket expenses.

Disclosures: Dr. Hayes reports grants from CNP Group LLC, other from MT Venom LLC, outside the submitted work.

\section{References}

1. Hayes WK, Mackessy SP. Sensationalistic journalism and tales of snakebite: are rattlesnakes rapidly evolving more toxic venom? Wilderness Environ Med. 2010;21:35-45.

2. Wingert W, Chan L. Rattlesnake bites in southern California and rationale for recommended treatment. West $J$ Med. 1988;148:37-44.

3. Hayes WK, Herbert SS, Bush SP, Rehling GC, Cardwell MD. Defensive bites by rattle snakes (genus Crotalus): venom expenditure, envenomation severity, and the importance of snake size. In: Program and Abstracts of the Biology of the Rattle snakes Symposium. Vol. 2. Loma Linda, CA: Loma Linda University; 2005:31. 
4. Janes DN, Bush SP, Kolluru GR. Large snake size suggests increased snakebite severity in patients bitten by rattlesnakes in southern California. Wilderness Environ Med. 2010;21:120-126.

5. Corbit AG, Hayes WK. Factors that influence the clinical severity of venomous snakebites in southern California. Toxicon. 2016;117:106.

6. Corbit AG. The dynamics of human and rattlesnake conflict in southern California [dissertation]. Loma Linda, CA: Loma Linda University; 2015.

7. Hayes WK. Ontogeny of striking, prey-handling and envenomation behavior of prairie rattlesnakes (Crotalus v. viridis). Toxicon. 1991;29:867-875.

8. Hayes WK. The snake venom-metering controversy: levels of analysis, assumptions, and evidence. In: Hayes WK, Beaman KR, Cardwell MD, Bush SP, eds. The Biology of Rattlesnakes. Loma Linda, CA: Loma Linda University Press; 2008:191-220.

9. Hayes WK, Herbert SS, Rehling GC, Gennaro JF. Factors that influence venom expenditure in viperids and other snake species during predatory and defensive contexts. In: Schuett GW, Höggren M, Douglas ME, Greene HW, eds. Biology of the Vipers. Eagle Mountain, UT: Eagle Mountain Publishing, LC; 2002:207-233.

10. de Roodt AR, Boyer LV, Lanari LC, et al. Venom yield and its relationship with body size and fang separation of pit vipers from Argentina. Toxicon. 2016;121:22-29.

11. McCollough NC, Gennaro JF. Treatment of venomous snakebite in the United States. Clin Toxicol. 1970;3:483-500.

12. Zamudio KR, Hardy DL, Martins M, Greene HW. Fang tip spread, puncture distance, and suction for snake bite. Toxicon. 2000;38:723-728.

13. Klauber L. A statistical study of the rattlesnakes. V. Head dimensions. Occas Papers San Diego Soc Nat Hist. 4:1-53.

14. Glaudas X, Jezkova T, Rodríguez-Robles JA. Feeding ecology of the Great Basin rattlesnake (Crotalus lutosus, Viperidae). Can J Zool. 2008;86:723-734.

15. Mitchell SW. Researches upon the venom of the rattlesnake: with an investigation of the anatomy and physiology of the organs concerned. Smithsonian Contributions to Knowledge. 1860:89-95.

16. Wüster W. Recent developments in rattlesnake phylogenetics, phylogeography, and species delimitation. In: Schuett GW, Feldner MJ, Smith CF, Reiserer RS, eds. The Rattlesnakes of Arizona Rodeo, NM: ECO Publishing; 2016:9-29.

17. Hardy DL, Greene HW. Surgery on rattlesnakes in the field for implantation of transmitters. Son Herpetol 1999;12:25-27.

18. Mertler CA, Vannatta RA. Advanced and Multivariate Statistical Methods: Practical Application and Interpretation 3rd ed. Los Angeles, CA: Pyrczak Publishing; 2004.

19. Cohen. Statistical Power Analysis for the Behavioral Sciences. 2nd ed. Hillsdale, NJ: Lawrence Erlbaum Associates; 1988.

20. Pierce CA, Block RA, Aguinis H. Cautionary note on reporting eta-squared values from multifactor ANOVA designs. Educ Psychol Meas. 2004;64:916-924.
21. Revell TK, Hayes WK. Desert iguanas (Dipsosaurus dorsalis) sleep less when in close proximity to a rattlesnake predator (Crotalus cerastes). J Herpetol. 2009;43:29-37.

22. Cundall D. Envenomation strategies, head form, and feeding ecology in vipers. In: Schuett GW, Höggren M, Douglas ME, Greene HW, eds. Biology of the Vipers. Eagle Mountain, UT: Eagle Mountain Publishing, LC; 2002:149-161.

23. Schmidt DF, Hayes WK, Hayes FE. Influence of prey movement on the aim of predatory strikes of the western rattlesnake (Crotalus viridis). Great Basin Nat. 1993;53:203-206.

24. Kardong KV. Kinesis of the jaw apparatus during swallowing in the cottonmouth snake, Agkistrodon piscivorus. Copeia. 1977;1977:338-348.

25. Cundall D, Deufel A. Influence of the venom delivery system on intraoral prey transport in snakes. Zool Anz. 2006;245:193-210.

26. Kardong KV. Kinesis of the jaw apparatus during the strike in the cottonmouth snake, Agkistrodon piscivorus. Forma Funct. 1974;7:327-354.

27. Duvall D, King MB, Gutzwiller KJ. Behavioral ecology and ethology of the prairie rattlesnake. Natl Geogr Res. 1985;1:80-111.

28. Gibbons JW, Dorcas ME. Defensive behavior of cottonmouths (Agkistrodon piscivorus) toward humans. Copeia. 2002;2002:195-198.

29. Beaman KR, Hayes WK. Annotated checklist of the rattlesnakes. In: Dreslik MJ, Hayes WK, Beaupre SJ, Mackessy SP, eds. The Biology of Rattlesnakes II. Rodeo, NM: ECO Herpetological Publishing and Distribution; 2017:13-21.

30. Pyron RA, Burbrink FT, Wiens JJ. A phylogeny and revised classification of Squamata, including 4161 species of lizards and snakes. BMC Evol Biol. 2013;13:93.

31. Reyes-Velasco J, Meik JM, Smith EN, Castoe TA. Phylogenetic relationships of the enigmatic longtailed rattlesnakes (Crotalus ericsmithi, C. lannomi, and C. stejnegeri). Mol Phylogenet Evol. 2013;69:524-534.

32. Russell FE. Snake venom poisoning in the United States. Annu Rev Med. 1980;31:247-259.

33. Blackman JR, Dillon S. Venomous snakebite: past, present, and future treatment options. J Am Board Fam Pract. 1992;5:399-405.

34. Shaw BA, Hosalkar HS. Rattlesnake bites in children: antivenin treatment and surgical indications. J Bone Joint Surg Am. 2002;84-A:1624-1629.

35. Nazim MH, Gupta S, Hashmi S, et al. Retrospective review of snake bite victims. WV Med J. 2008;104:30-34.

36. Lawrence WT, Giannopoulos A, Hansen A. Pit viper bites: rational management in locales in which copperheads and cottonmouths predominate. Ann Plast Surg. 1996;36:276285.

37. Herbert SS, Hayes WK. Denim clothing reduces venom expenditure by rattlesnakes striking defensively at model human limbs. Ann Emerg Med. 2009;54:830-836.

38. Nishioka SDA, Silveira P, Bauab F, Simon J. Bite marks are useful for the differential diagnosis of snakebite in Brazil. Wilderness Environ Med. 1995;6:183-188. 